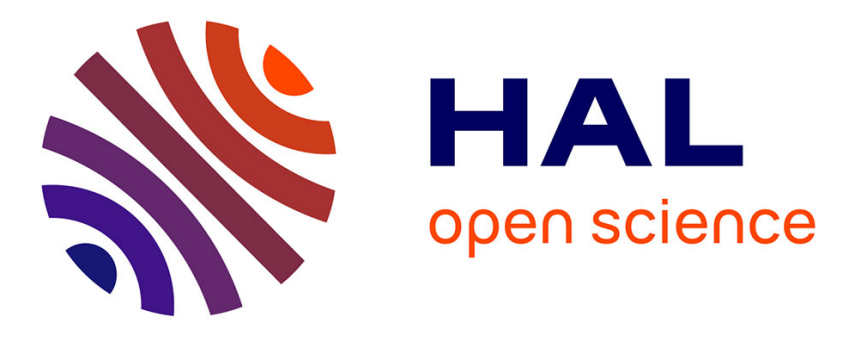

\title{
Sparse Reconstruction Algorithms for Nonlinear Microwave Imaging
}

Hidayet Zaimaga, Aurélia Fraysse, Marc Lambert

\section{To cite this version:}

Hidayet Zaimaga, Aurélia Fraysse, Marc Lambert. Sparse Reconstruction Algorithms for Nonlinear Microwave Imaging. 25th European Signal Processing Conference (EUSIPCO 2017), Aug 2017, Kos island, Greece. pp.743-747, 10.23919/eusipco.2017.8081300 . hal-01587676

\section{HAL Id: hal-01587676 \\ https://hal-centralesupelec.archives-ouvertes.fr/hal-01587676}

Submitted on 15 Sep 2017

HAL is a multi-disciplinary open access archive for the deposit and dissemination of scientific research documents, whether they are published or not. The documents may come from teaching and research institutions in France or abroad, or from public or private research centers.
L'archive ouverte pluridisciplinaire $\mathbf{H A L}$, est destinée au dépôt et à la diffusion de documents scientifiques de niveau recherche, publiés ou non, émanant des établissements d'enseignement et de recherche français ou étrangers, des laboratoires publics ou privés. 


\title{
Sparse Reconstruction Algorithms for Nonlinear Microwave Imaging
}

\author{
Hidayet Zaimaga \\ Laboratoire des Signaux et Systèmes \\ CNRS UMR8506 \\ CentraleSupélec-Univ.Paris-Sud
}

\author{
Aurélia Fraysse \\ Laboratoire des Signaux et Systèmes \\ CNRS UMR8506 \\ CentraleSupélec-Univ.Paris-Sud
}

\author{
Marc Lambert \\ Group of electrical engineering - Paris, \\ CNRS UMR8507, \\ CentraleSupélec, Univ. Paris-Sud, \\ Université Paris-Saclay, \\ Sorbonne Universités, UPMC Univ Paris 06
}

\begin{abstract}
This paper presents a two-step inverse process which allows sparse recovery of the unknown (complex) dielectric profiles of scatterers for nonlinear microwave imaging. The proposed approach is applied to a nonlinear inverse scattering problem arising in microwave imaging and correlated with joint sparsity which gives multiple sparse solutions that share a common nonzero support. Numerical results demonstrate the potential of the proposed two step inversion approach when compared to existing sparse recovery algorithm when considering small scatterers.
\end{abstract}

\section{INTRODUCTION}

The main goal of microwave imaging is to estimate the location of scatterers in a region of interest by retrieving the distribution of the dielectric properties. Development of efficient methods and techniques for solving such an inverse electromagnetic scattering problem has been attracting significant research interest around the world due to its potential in diverse applications such as material characterization, subsurface prospecting, remote sensing, and non-destructive testing and evaluation [1], [2].

Inverse electromagnetic scattering problems deal with the reconstruction of the contrast field profile of an imaged object. However, implementation of stable, reliable, and efficient reconstruction algorithms in this context is challenging because of the nonlinearity and ill-posedness of the problem to be solved [1], [3], [4]. To circumvent the non-linearity or its effects several methods including global optimization tools, multi-step information retrieval techniques, and qualitative methods have been introduced. Moreover, first-order approximations such as diffraction tomography, Born and Rytov approximations have been introduced to linearize the problem [1], [3]. However, these methods are limited to investigation domains involving weak scatterers and produce a non-negligible error in the reconstruction when applied to configurations in which the weakness of the scatterers is no longer verified.

On the other hand, sparsity-promoting regularization has become an interesting alternative to overcome the non-uniqueness and/or numerical instability of the inversion process [5], [6] in recent years. The reason behind this is that many images have sparse representations with respect to their expansion basis and this yields new developing approaches that minimize the cost functions with zeroth/first norm penalty terms to enforce sparsity using highly effective iterative shrinkage algorithms. Such an increased interest is proven by the number of publications in broad domains [4]-[9]. In this framework, sparsity promoting regularized approaches assume that the unknown functions can be expressed as a sparse set of coefficients with respect to an appropriate basis. In recent years, sparse methods for microwave imaging have been developed, see [10], [11], using a linear approximation of the scattered signal. One of the main contributions of the proposed paper is that it is based on joint sparsity. The principal interest of such a method is to accurately reconstruct the unknown scatterers without linear approximation and present an efficient recovery algorithm of sparse scatterers by reducing possible ambiguities on the scatterer sparsity deduced from the null values of the equivalent current [1], [12]. This new approach will be compared to our previous work where the nonlinear inverse problem is solved by directly enforcing the sparsity on the unknown contrast [13].

In recent years, the problem of joint sparsity regularization has been studied in various areas (multi-task learning, group Lasso [14], distributed compressive sensing, etc.) [15], [16]. Jointly sparse solutions have a common nonzero support and the jointly sparse based model takes into account any additional information about the structure of the solutions more than just sparsity approaches [15].

The paper is structured as follows. The electromagnetic scattering model is briefly recalled in Section II-A. Section III deals with our proposed imaging method based on the sparsity minimization. Section IV provides numerical examples in order to assess the features and limitations of the proposed approach while comparing it with the method suggested in [13]. Finally, some conclusions are reported in Section V.

\section{Problem Statement}

\section{A. Formulation}

Let us consider the scenario as in Fig.1 with reference to a time-harmonic two-dimensional Transverse Magnetic (TM) problem. A given source generates an incident electric field $E^{\text {inc }}$ which is polarized along the $z$-axis with implicit time factor 


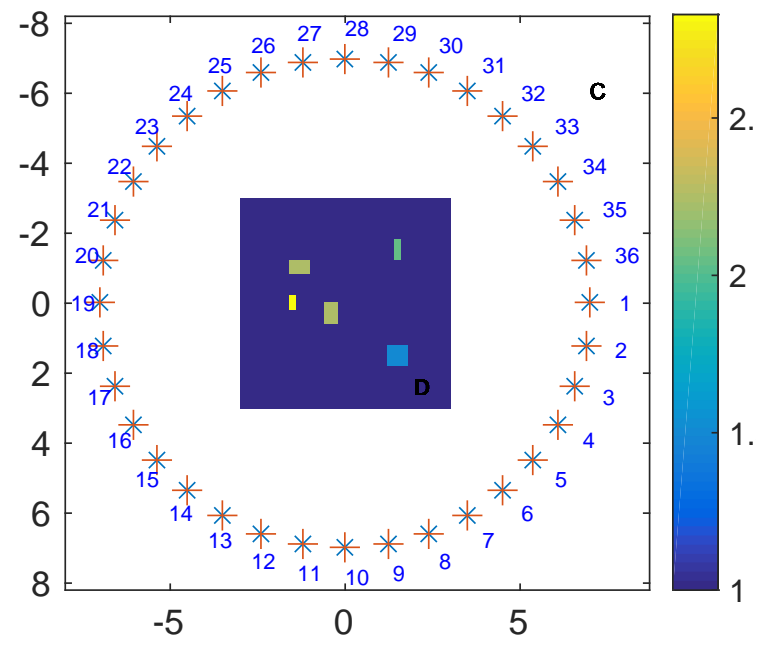

(a) Configuration 1

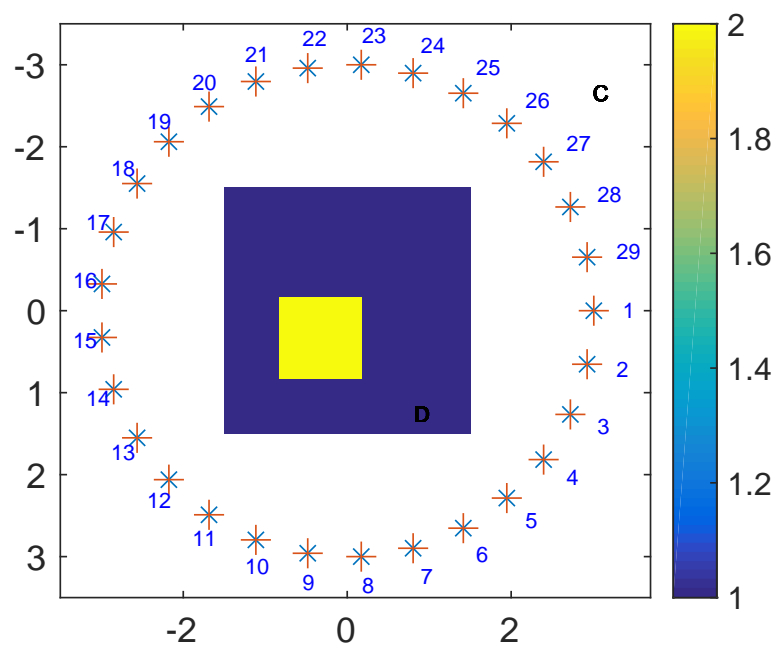

(b) Configuration 2

Fig. 1: Measured configuration of actual permittivity profile and source-receiver locations on $(x(\mathrm{~m}), y(\mathrm{~m}))$ axis.

$\exp (-\mathrm{i} \omega t)$ where $\omega$ is the angular frequency. The object is considered in an investigation domain $D$ and the different media are characterized by their propagation constant $k(\mathbf{r})$ such that $k(\mathbf{r})^{2}=\omega^{2} \varepsilon_{0} \varepsilon_{r}(\mathbf{r}) \mu_{0}+\mathrm{i} \omega \mu_{0} \sigma(\mathbf{r})$, where $\varepsilon_{0}$ and $\mu_{0}$ are the permittivity and the permeability of air respectively, $\varepsilon_{r}(\mathbf{r})$ and $\sigma(\mathbf{r})$ are the relative permittivity and conductivity of the medium as $\mathbf{r} \in D$ is an observation point. The dielectric properties of $D$ are described by the inhomogeneous contrast function defined as $\chi(\mathbf{r})=\left(k(\mathbf{r})^{2}-k_{B}^{2}\right)$, where $k_{B}^{2}=\omega^{2} \varepsilon_{0} \mu_{0}$ is the propagation constant of the embedding medium. We assume that sources and receivers are located at positions $\mathbf{r}_{i}$ and $\mathbf{r}_{r}$ respectively.

The scattered electric field $E^{\text {diff }}\left(\mathbf{r}_{r}, \mathbf{r}_{i}\right)$ measured by a receiver placed at $\mathbf{r}_{r}$ due to the incident wave emitted by a source placed at $\mathbf{r}_{i}$ adheres to the following integral equation [2]

$$
E^{\operatorname{diff}}\left(\mathbf{r}_{r}, \mathbf{r}_{i}\right)=\int_{D} G\left(\mathbf{r}_{r}, \mathbf{r}^{\prime}\right) J\left(\mathbf{r}^{\prime}, \mathbf{r}_{i}\right) d \mathbf{r}^{\prime}
$$

with

$$
J\left(\mathbf{r}, \mathbf{r}_{i}\right)=\chi(\mathbf{r}) E\left(\mathbf{r}, \mathbf{r}_{i}\right)
$$

where $J\left(\mathbf{r}, \mathbf{r}_{i}\right)$ and $E\left(\mathbf{r}, \mathbf{r}_{i}\right)$ are the equivalent current and the total electric field respectively, both induced within the object by the incident wave, $G\left(\mathbf{r}, \mathbf{r}^{\prime}\right)=\frac{-1}{4 \omega \varepsilon_{0}} \mathrm{H}_{0}^{(1)}\left(k_{B}\left\|\mathbf{r}-\mathbf{r}^{\prime}\right\|\right)$ and $\mathrm{H}_{0}^{(1)}$ is the 0 -th order Hankel function of the first kind. Furthermore $E\left(\mathbf{r}, \mathbf{r}_{i}\right)$ is obtained as

$$
E\left(\mathbf{r}, \mathbf{r}_{i}\right)=E^{\mathrm{inc}}\left(\mathbf{r}, \mathbf{r}_{i}\right)+\int_{D} G\left(\mathbf{r}, \mathbf{r}^{\prime}\right) J\left(\mathbf{r}^{\prime}, \mathbf{r}_{i}\right) d \mathbf{r}^{\prime} \forall \mathbf{r} \in D .
$$

The direct problem is defined as the calculation of $E^{\text {diff }}\left(\mathbf{r}_{r}, \mathbf{r}_{i}\right)$ by solving (1) and (3) when $\chi(\mathbf{r}), G\left(\mathbf{r}, \mathbf{r}^{\prime}\right)$ and $E^{\text {inc }}\left(\mathbf{r}, \mathbf{r}_{i}\right)$ are known whereas the inverse problem (or imaging problem) is defined as the determination of $\chi(\mathbf{r})$ within a prescribed domain $D$ from the knowledge of $E^{\text {diff }}\left(\mathbf{r}_{r}, \mathbf{r}_{i}\right), G\left(\mathbf{r}, \mathbf{r}^{\prime}\right)$ and $E^{\text {inc }}\left(\mathbf{r}, \mathbf{r}_{i}\right)$ for $N_{s}$ sources and $N_{r}$ receivers.

\section{B. Discretization of the problem}

Thanks to a classical method of moments using pulse basis/point matching method [17] where the domain under test $D$ is discretized into $N=N_{x} \times N_{y}$ pixels, a discretized version of the previous equations is obtained. In so doing the discretized version of (1) stands as

$$
\mathbf{E}_{i}^{\text {diff }}=\mathbf{G}_{\mathbf{o r}} \mathbf{J}_{i}, \quad i=1, \ldots, N_{s}
$$

where $\mathbf{E}_{i}^{\text {diff }}$ and $\mathbf{J}_{i}$ are complex vectors of size $N_{r}$ and $N$ respectively and $\mathbf{G}_{\text {or }}$ a complex matrix of size $N_{r} \times N$. The discretized version of (2) is then

$$
\mathbf{J}_{i}=\operatorname{diag}(\chi) \mathbf{E}_{i}, \quad i=1, \ldots, N_{s},
$$

where $\mathbf{E}_{i}$ and $\chi$ are complex vectors of size $N$ and $\operatorname{diag}(\chi)$ is a diagonal matrix of size $N \times N$ obtained from $\chi$. Finally the discretized version of (3) is

$$
\mathbf{E}_{i}=\mathbf{E}_{i}^{\mathrm{inc}}+\mathbf{G}_{\mathbf{o o}} \mathbf{J}_{i}, \quad i=1, \ldots, N_{s}
$$


where $\mathbf{E}_{i}^{\text {inc }}$ is a complex vector of size $N$ and $\mathbf{G}_{\text {oo }}$ a matrix of size $N \times N$.

By combining (4) and (6) and using $\mathbf{J}_{i}^{\mathrm{inc}}=\operatorname{diag}(\chi) \mathbf{E}_{i}^{\mathrm{inc}} i=1, \ldots, N_{s}$ the inverse problem can be rewritten as

$$
\chi^{\star}=\underset{\chi}{\operatorname{argmin}}\left\|\boldsymbol{\zeta}_{i}-\mathbf{G}_{\mathbf{o r}} \operatorname{diag}(\boldsymbol{\chi})\left[\mathbf{I}-\operatorname{diag}(\boldsymbol{\chi}) \mathbf{G}_{\mathbf{o o}}\right]^{-1} \mathbf{J}_{i}^{\mathrm{inc}}\right\|_{2}
$$

where $\zeta_{i}$ is a vector of size $N_{r}$ which gathered the signal due to the source $\# i$ measured by the $N_{r}$ receivers. It can be seen from (7) that the inverse problem is nonlinear in $\chi$ and ill-posed.

\section{Two-STEP INVERSion Procedure}

Instead of directly solving the nonlinear inverse problem given by (7) as we did in [13], we adopt a two-step method which firstly consists of finding the equivalent current $\mathbf{J}_{i}$ for $i=1, \ldots, N_{s}$ using (1) and then looking for $\chi$ using the combination of (2) and (3). This approach leads us to have two linear minimization problems solved by exploiting the jointly-sparse aspect of the sought equivalent currents solution of the first step and a classical $l^{2}$-minimization of a linear problem for the second step.

\section{A. First step: reconstruction of the equivalent currents}

The following optimization problem is solved

$$
\mathbf{J}_{i}^{\star}=\underset{\mathbf{J}_{i}}{\operatorname{argmin}}\left[\frac{1}{2}\left\|\boldsymbol{\zeta}_{i}-\mathbf{G}_{\mathbf{o r}} \mathbf{J}_{i}\right\|_{2}\right] \quad i=1, \cdots, N_{s} .
$$

where $\boldsymbol{\zeta}_{i}$ is a vector of size $N_{r}$ which gathered the signal due to the source \#i measured by the $N_{r}$ receivers.

The main idea of the approach is to take into account that, as shown by (5), $\mathbf{J}_{i}$ and $\chi$ share the same support, which means that when $\chi^{j}=0$ then $J_{i}^{j}=0, \forall i=1, \ldots, N_{i}$ where $\chi^{j}$ and $J_{i}^{j}$ are the $j$ element of $\chi$ and $\mathbf{J}_{i}$ respectively. Taking into account this hypothesis, (8) can be recast as a minimization problem under the constraints that $\mathbf{J}_{i}$ has the same sparse support for each source $i$, leading to the use of a (weighted) $l_{2,1}$-regularization to enforce joint sparsity such as

$$
\min _{\mathbf{J}}\|\mathbf{J}\|_{w, 2,1}:=\sum_{i=1}^{N} w_{i}\left\|\mathbf{J}^{i}\right\|_{2} \quad \text { s. t. } \quad \mathbf{G}_{\mathbf{o r}} \mathbf{J}_{i}=\boldsymbol{\zeta}_{i}
$$

for all $i=1, \cdots, N_{s}$ where $\mathbf{J}^{i}$ denotes the $i$-th row of $\mathbf{J}$ while $w_{i}$ is corresponding with the weight [15]. (9) is solved using the YALL1 Group [15] package which encodes the joint sparsity model.

\section{B. Second step: reconstruction of the contrast function}

Once $\mathbf{J}_{i}$ is known, the contrast function is obtained by solving the following minimization problem [18]

$$
\boldsymbol{\chi}^{\star}=\operatorname{argmin} \sum_{i=1}^{N_{s}}\left\|\mathbf{J}_{i}-\operatorname{diag}(\boldsymbol{\chi}) \mathbf{E}_{i}\right\|_{2}=\frac{\sum_{i=1}^{N_{s}} \mathbf{J}_{i} \cdot \overline{\mathbf{E}}_{i}}{\sum_{i=1}^{N_{s}} \mathbf{E}_{i} \cdot \overline{\mathbf{E}}_{i}},
$$

where $\mathbf{E}_{i}$ has been obtained using (6) and $\overline{\mathbf{E}}_{i}$ being its conjugate.

\section{Numerical Results}

In this section we consider two examples in order to present our proposed method and compare it with the method based on Soft Shrinkage algorithm proposed in [13]. The frequency of the emitting wave is $f=300 \mathrm{MHz}$ and the measured field samples are generated by adding $10 \mathrm{~dB}$ white Gaussian noise. The various obstacles are embedded in air. The corresponding wavelength in air is given by $\lambda=\frac{2 \pi}{k_{B}}$. The direct and inverse problems are solved with different discretization in order to avoid the so-called "inverse crime", while $N_{x}$ and $N_{y}$ are the number of pixels along the $x$ and $y$ direction respectively.

- First example (Fig. 1a): Five objects of physical characteristics described in Tab. I are embedded in a $l=6 \lambda$-sided square investigation domain $D$. The discretization size is $N_{x}=N_{y}=80$ for the forward problem and $N_{x}=N_{y}=30$ for the inverse problem. The number of transmitters and receivers located around the investigation area is $N_{s}=N_{r}=36$ evenly distributed on a circle of radius $r=7 \lambda$.

- Second example (Fig. 1b): A single square obstacle of $\lambda$-side length is embedded in a of $l=3 \lambda$-sided square investigation domain $D$, the direct problem being solved using $N_{x}=N_{y}=36$ pixels and the inverse problems with $N_{x}=N_{y}=18$. The number of transmitters and receivers located around the investigation area is $N_{s}=N_{r}=29$ evenly distributed on a circle of radius $r=3 \lambda$.

We choose the primal-based solver where the linear system is exactly solved for the parameters of YALL1 algorithm. The weights have been chosen as $w_{i}=1(i=1, \cdots, N)$ and the initialization is $\mathbf{J} \equiv 0$ (no prior information). 
TABLE I: Description of the five obstacles, $x, y$ being the coordinate of the center of the obstacle (in $\mathrm{m}$ ), $L_{x}, L_{y}$ its lengths (in $\mathrm{m}$ ) and $\varepsilon_{r}$ and $\sigma$ its relative permittivity and conductivity (the latter in $\mathrm{S} \mathrm{m}^{-1}$ )

\begin{tabular}{ccccllc}
\hline$\#$ & $x$ & $y$ & $L_{x}$ & $L_{y}$ & $\varepsilon_{r}$ & $\sigma$ \\
\hline 1 & -1.5 & -1.5 & 0.5 & 0.5 & 1.5 & 0.0022 \\
2 & -0.33 & 0.44 & 0.5 & 0.5 & 2.25 & 0 \\
3 & 1.5 & -1.5 & 0.5 & 0.33 & 2 & 0.00445 \\
4 & 0 & 1.5 & 0.5 & 0.33 & 2 & 0.02225 \\
5 & 1 & 1.33 & 0.5 & 0.5 & 1 & 0.02225 \\
\hline
\end{tabular}
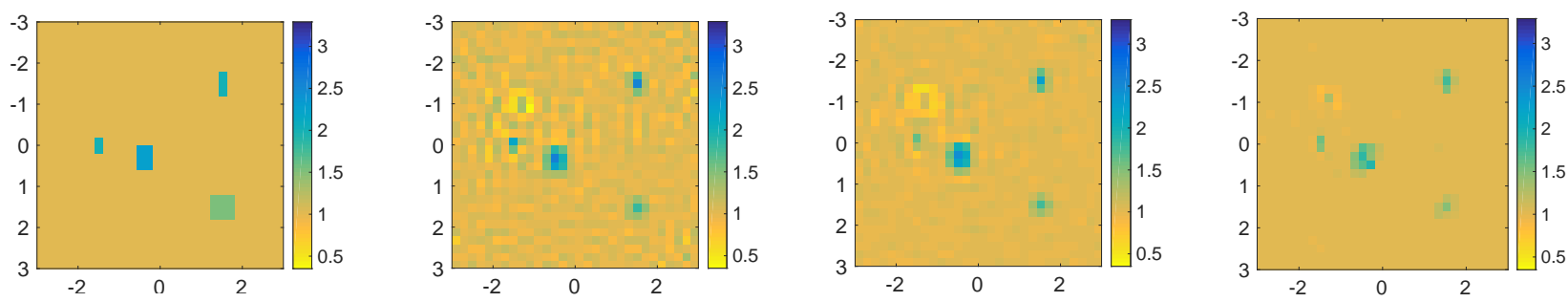

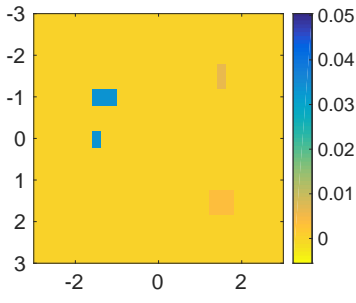

(a) Exact

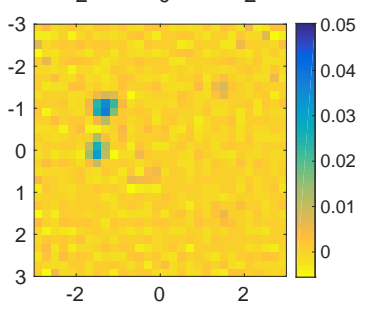

(b) Soft Shrinkage Alg. $\alpha=1 \times 10^{-6}$

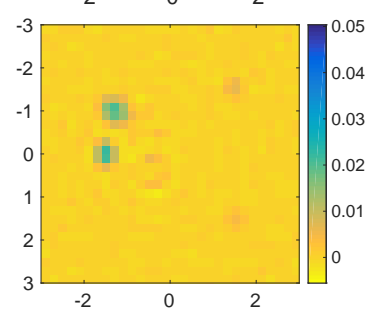

(c) Soft Shrinkage Alg $\alpha=5 \times 10^{-3}$

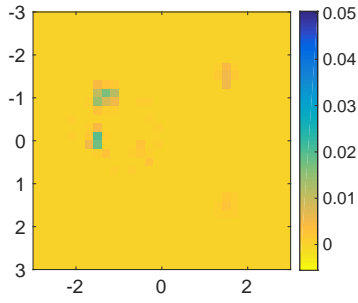

(d) Jointly-sparse model

Fig. 2: Retrieval of permittivity ( $\varepsilon_{r}$, top) and conductivity ( $\sigma$, bottom) by using sparsity with $10 \mathrm{~dB}$ noise data.

The relative error norm on $\chi$ as a comparison criterion is expressed as

$$
\operatorname{err}_{\chi}=\frac{\operatorname{Tr}\left(\left\|\operatorname{diag}(\boldsymbol{\chi})-\operatorname{diag}\left(\boldsymbol{\chi}^{\text {true }}\right)\right\|_{2}\right)}{\operatorname{Tr}\left(\left\|\operatorname{diag}\left(\boldsymbol{\chi}^{\text {true }}\right)\right\|_{2}\right)},
$$

where $\chi^{\text {true }}$ is the true contrast to be found and $\operatorname{Tr}(\mathbf{A})$ stands for the trace of the matrix A. The discrepancy between the model and the measurement is as following:

$$
\operatorname{err}_{\mathbf{E}^{\mathrm{diff}}}=\frac{\sum_{i=1}^{N_{i}}\left\|\boldsymbol{\zeta}_{i}-\mathbf{E}_{i}^{\mathrm{diff}}(\chi)\right\|_{2}}{\sum_{i=1}^{N_{i}}\left\|\boldsymbol{\zeta}_{i}\right\|_{2}},
$$

where $\mathbf{E}^{\text {diff }}(\chi)$ is computed by solving the direct problem with the reconstructed $\chi$.

It is worthwhile to mention that sparseness is a relative concept with respect to a basis [1]. In the case of a pixel basis, a fast way to estimate the sparsity of our problem is to define it as the ratio of the obstacle's areas to the investigation domain $D$ area. Following this, the sparseness of the first example is around $3 \%$ whereas the one of the second example is around $11 \%$. One of the key points of our examples is that in the first one the scatterers are sparse with respect to their expansion basis while in the second one the scatterer is less sparse and our proposed approach in this paper performs differently for these two cases.

It can be observed that when we have sparse scatterers (as in Fig. 1a) the two-step inversion approach gives better reconstruction in terms of quality of $\chi$ and processing time compared to the soft shrinkage algorithm as shown in Tab. II. On the other hand, even if the two-step inversion approach is faster in the reconstruction of the scatterer in Fig. 1b, soft shrinkage algorithm achieves a better reconstruction quality with an appropriate regularization parameter.

\section{CONCLUSION}

A two-step inversion approach has been proposed in order to solve a nonlinear inverse problem by applying joint sparsity to get the equivalent current, then the unknown contrast. Furthermore, the proposed approach has been compared with the approach that directly yields the contrast through enforcement of sparsity by soft shrinkage thresholding. Both approaches produce sharp and good reconstruction of dielectric profiles in sparse domains and keep their convergence during the reconstruction. 

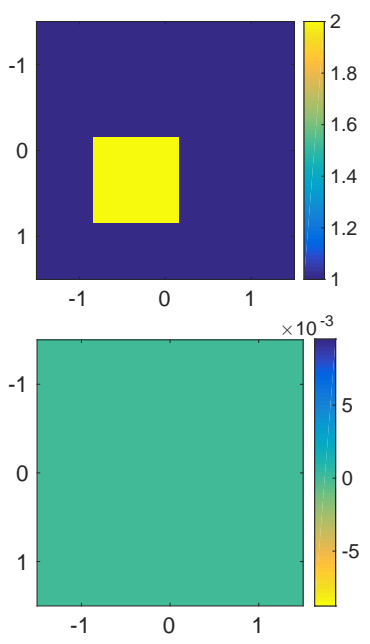

(a) Exact
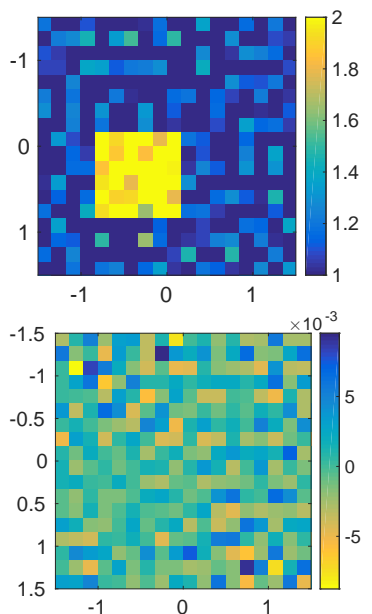

(b) Soft Shrinkage Alg. $\alpha=1 \times 10^{-6}$
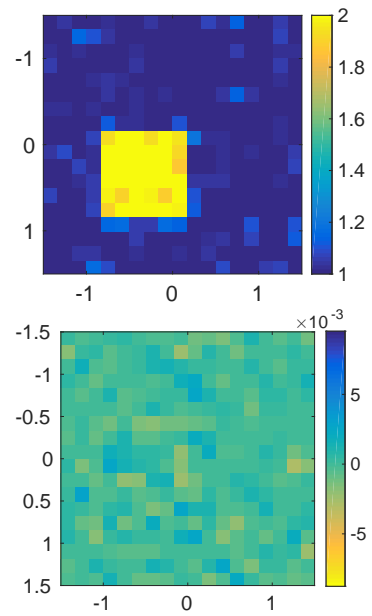

(c) Soft Shrinkage Alg. $\alpha=2.5 \times 10^{-2}$
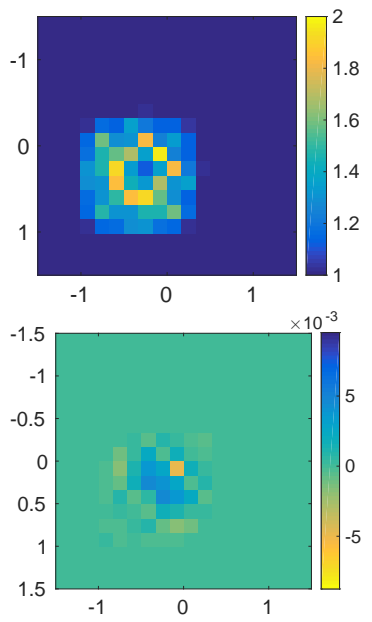

(d) Jointly-sparse model

Fig. 3: Retrieval of permittivity ( $\varepsilon_{r}$, top) and conductivity ( $\sigma$, bottom) by using sparsity with $10 \mathrm{~dB}$ noise data.

TABLE II: Error and average simulation time in seconds for the proposed methods

\begin{tabular}{l|lccc}
\hline Scatterer & Scenario & Time $(\mathrm{s})$ & $\operatorname{err}_{\chi}$ & $\operatorname{err}_{\mathbf{E}^{\text {diff }}}$ \\
\hline \multirow{3}{*}{ Fig. 1a } & Without sparsity & 367.1 & 0.1754 & 0.1350 \\
& Soft Shrinkage Alg. & 131.8 & 0.1413 & 0.1206 \\
& Jointly-sparse model & $\mathbf{2 5 . 1}$ & $\mathbf{0 . 1 3 7 5}$ & 0.1995 \\
\hline \multirow{3}{*}{ Fig. 1b } & Without sparsity & 64.5 & 0.2527 & 0.1045 \\
& Soft Shrinkage Alg. & 16.2 & $\mathbf{0 . 0 6 3 1}$ & 0.0790 \\
& Jointly-sparse model & $\mathbf{8 . 3}$ & 0.1720 & 0.5684 \\
\hline
\end{tabular}

This method as proposed consumes less time and ensures better imaging quality compared to an iterative method with soft thresholding in the reconstruction of sparse scatterers as exhibited in Tab.II.

Even though the results are promising and advantageous, a distinctive feature of the proposed methods is to use the wavelet transform in order to expand the contrast function characterizing scatterers with respect to the surrounding medium and advance inversion reliability [19], [20].

\section{ACKNOWLEDGMENT}

This work is supported by DIGITEO France through the SIRENA project (2014-2017) under the "Call for Chairs 2014."

\section{REFERENCES}

[1] L. Poli, G. Oliveri, and A. Massa, "Microwave imaging within the first-order Born approximation by means of the contrast-field Bayesian compressive sensing," IEEE Trans. Antennas Propagat., vol. 60, no. 6, pp. 2865-2879, 2012.

[2] M. Pastorino, Microwave Imaging. John Wiley \& Sons, 2010, vol. 208.

[3] F. Di Benedetto, C. Estatico, J. G. Nagy, and M. Pastorino, "Numerical linear algebra for nonlinear microwave imaging," Electron. Trans. Numer. Anal., vol. 33, pp. 105-125, 2009.

[4] R. Ramlau and G. Teschke, "A Tikhonov-based projection iteration for nonlinear ill-posed problems with sparsity constraints," Numer. Math., vol. 104, no. 2, pp. 177-203, 2006.

[5] I. Daubechies, M. Defrise, and C. De Mol, "An iterative thresholding algorithm for linear inverse problems with a sparsity constraint," arXiv preprint math/0307152, 2003.

[6] B. Jin and P. Maass, "Sparsity regularization for parameter identification problems," Inverse Prob., vol. 28, no. 12, p. 123001, 2012.

[7] M. Grasmair, M. Haltmeier, and O. Scherzer, "Sparse regularization with lq penalty term," Inverse Prob., vol. 24, no. 5, p. 055020, 2008

[8] M. Hanke, A. Neubauer, and O. Scherzer, "A convergence analysis of the Landweber iteration for nonlinear ill-posed problems," Numer. Math., vol. 72, no. 1, pp. 21-37, 1995.

[9] A. Desmal and H. Bagci, "Sparse electromagnetic imaging using nonlinear Landweber iterations," Prog. Electromagn. Res., 2015.

[10] S. Jun, Z. Xiaoling, X. Gao, and J. Jianyu, "Signal processing for microwave array imaging: Tdc and sparse recovery," IEEE Trans. Geosci. Remote Sensing, vol. 50, no. 11, pp. 4584-4598, 2012.

[11] S.-J. Wei, X.-L. Zhang, J. Shi, and K.-F. Liao, "Sparse array microwave 3-d imaging: Compressed sensing recovery and experimental study," Prog. Electromagn. Res., vol. 135, pp. 161-181, 2013

[12] E. J. Candès and M. B. Wakin, "An introduction to compressive sampling," IEEE Signal Processing Mag., vol. 25, no. 2, pp. 21-30, 2008.

[13] H. Zaimaga and M. Lambert, "Sparsity-enforced microwave inverse scattering using soft shrinkage thresholding," in European Signal Processing Conference (EUSIPCO 2016), Budapest, Hungary, pp. 350-354. 
[14] M. Yuan and Y. Lin, "Model selection and estimation in regression with grouped variables," J R Stat Soc Series B Stat Methodol., vol. 68, no. 1, pp. 49-67, 2006.

[15] W. Deng, W. Yin, and Y. Zhang, "Group sparse optimization by alternating direction method," in SPIE Optical Engineering+ Applications. International Society for Optics and Photonics, 2013, pp. 88 580R-88 580R-15.

[16] D. Baron, M. F. Duarte, M. B. Wakin, S. Sarvotham, and R. G. Baraniuk, "Distributed compressive sensing," arXiv preprint arXiv:0901.3403, 2009.

[17] J. Richmond, "Scattering by a dielectric cylinder of arbitrary cross section shape," IEEE Trans. Antennas Propagat., vol. 13, no. 3, pp. 334-341, May 1965.

[18] A. Abubakar and P. M. van den Berg, "Iterative forward and inverse algorithms based on domain integral equations for three-dimensional electric and magnetic objects," J. Comput. Phys., vol. 195, no. 1, pp. 236-262, 2004.

[19] O. M. Bucci, L. Crocco, T. Isernia, and V. Pascazio, "Subsurface inverse scattering problems: quantifying, qualifying, and achieving the available information," IEEE Trans. Geosci. Remote Sensing, vol. 39, no. 11, pp. 2527-2538, 2001

[20] S. G. Mallat, "A theory for multiresolution signal decomposition: the wavelet representation," IEEE Trans. Pattern Anal. Machine Intell., vol. 11, no. 7, pp. 674-693, 1989. 